\title{
DERIVED AND INTEGRAL SETS OF BASIC SETS OF POLYNOMIALS
}

M. N. MIKHAIL

For the general terminology used in this paper the reader is referred to Whittaker's books $[2 ; 3]$. I shall investigate the mode of increase and the effectiveness of basic derived and integral sets of any finite order. So far as I know, this is done here for the first time.

Let $p_{n}(z)=\sum_{i} p_{n i} z^{i}, q_{n}(z)=\sum_{i} q_{n i} z^{i}$ be two basic sets; then the product set $\left\{u_{n}(z)\right\}=\left\{p_{n}(z)\right\}\left\{q_{n}(z)\right\}$, in this order, is defined by $u_{i j}=\sum_{h} p_{i h} q_{h j}$. We have $z^{n}=\sum_{i} \pi_{n i} p_{i}(z)=\sum_{i} \lambda_{n i} q_{i}(z)$.

It follows from the definition of a basic set of polynomials that the first derived set: $p_{0}^{\prime}(z), p_{1}^{\prime}(z), p_{2}^{\prime}(z), \cdots$ form a basic set of polynomials with, perhaps, the omission of one of the polynomials. Thus the $h$ th derived set $\left\{D^{h}\left\{p_{n}(z)\right\}\right\}$ will form a basic set $\left\{v_{n}(z)\right\}$, if through differentiation of the basic set $\left\{p_{n}(z)\right\}$ certain polynomials, at most $h$ of these, are omitted.

Also, $1, \int_{c_{0}}^{z} p_{0}(x) d x, \int_{c_{1}}^{z} p_{1}(x) d x, \cdots$ form a basic set of polynomials, where $c_{0}, c_{1}, \cdots$ are any constants. Thus the $h$ th integral set $\left\{D^{-h}\left\{p_{n}(z)\right\}\right\}$ will form with additional polynomials: 1, z, $z^{2} / 2 !, \cdots, z^{h-1} /(h-1) !$ a basic set $\left\{t_{n}(z)\right\}$.

I shall use an auxiliary simple basic set $\left\{Q_{n}(z)\right\}$ in which $Q_{n}(z)=z^{n}$ for all $n$. $Q$ 's and $\beta$ 's are the coefficients and operators in $\left\{Q_{n}(z)\right\}$, its derived and its integral sets.

Lemma 1. If $\left\{p_{n}(z)\right\}$ is a basic set of polynomials, then

$$
\left\{p_{n}(z)\right\} \equiv\left\{p_{n}(z)\right\}\left\{Q_{n}(z)\right\} \text {. }
$$

Lemma 2. $\left\{D^{h}\left\{p_{n}(z)\right\}\right\} \equiv\left\{p_{n}(z)\right\}\left\{D^{h}\left\{Q_{n}(z)\right\}\right\}$.

PROOF. Let $\left\{v_{n-h}(z)\right\} \equiv\left\{D^{h}\left\{p_{n}(z)\right\}\right\}$ (see introduction), then we have

$$
\begin{aligned}
v_{n-h}(z) & =\sum_{i} p_{n i}\left(D^{h}\left(Q_{i}(z)\right)\right. \\
& =\sum_{i} p_{n i} \cdot i(i-1) \cdots(i-h+1) z^{i-h}=D^{h}\left(p_{n}(z)\right),
\end{aligned}
$$

whence the required result.

N.B. In what follows, I shall take 0 as the lower limit of integration for every integral set.

Received by the editors June 2, 1952. 
LEMма 3. If we define the integral set:

$$
\left\{t_{n+1}(z)\right\} \equiv\left\{D^{-1}\left\{p_{n}(z)\right\}\right\} \equiv\left\{\int_{0}^{z} p_{n}(x) d x\right\}
$$

it follows that

$$
\left\{D^{-h}\left\{p_{n}(z)\right\}\right\} \equiv\left\{p_{n}(z)\right\}\left\{D^{-h}\left\{Q_{n}(z)\right\}\right\} .
$$

Proof. We have

$$
\begin{aligned}
t_{n+h}(z) & =\sum_{i} p_{n i}\left(D^{-h}\left(Q_{i}(z)\right)\right) \\
& =\sum_{i} p_{n i} \frac{1}{(i+1)(i+2) \cdots(i+h)} z^{i+h}=D^{-h}\left(p_{n}(z)\right),
\end{aligned}
$$

whence the required result.

N.B. As mentioned before, $\left\{D^{-h}\left\{p_{n}(z)\right\}\right\}$ is not a basic set, but it can be made basic by the addition of the polynomials: $1, z, z^{2}, \cdots$, $z^{h-1}$. These added polynomials (finite in number) have no effect on the properties of the set. The method of examining the derived set or the integral set as a product, combined with my previous results [1], yields a number of results about effectiveness and order of the new set. Hence I state the following theorems:

THEOREM 1. $\left\{p_{n}(z)\right\}$ is a basic set of polynomials, such that $\lim \sup _{n \rightarrow \infty} D_{n} / n=a(a \geqq 1)$. Then its hth basic derived set is effective in a domain which either contains the domain of effectiveness of the original set or is the same, the outer radius of the domain being always the same.

Proof. By Lemma 2, $\left\{D^{n}\left\{p_{n}(z)\right\}\right\} \equiv\left\{p_{n}(z)\right\}\left\{D^{h}\left\{Q_{n}(z)\right\}\right\}$.

The following definitions were introduced in [1].

Let $s(n)$ be a sequence of integers such that

$$
0 \leqq s(n)<n, \quad \lim _{n \rightarrow \infty} s(n) / n=\sigma<1 .
$$

Let $d(n)$ be a sequence of integers such that

$$
0<d(n)<D_{n}, \quad \lim _{n \rightarrow \infty} d(n) / n=1 .
$$

Let $g(n)$ be a sequence of integers such that

$$
n<g(n) \leqq D_{n}, \quad \lim _{n \rightarrow \infty} g(n) / n=\sigma^{\prime}>1 .
$$

Put

$$
\sup _{i}\left|\pi_{n i}\right|\left|p_{i s(n)}\right|=b(n, s(n)),
$$




$$
\begin{aligned}
\limsup _{n \rightarrow \infty}\{b(n, s(n))\}^{1 /\{n-s(n)\}} & =b(s(n)), \\
\sup _{s(n)} b(s(n)) & =b ; \\
\sup _{\bullet}\left|\pi_{n i}\right|\left|p_{i} d(n)\right| & =C(n, d(n)), \\
\limsup _{n \rightarrow \infty}\{C(n, d(n))\}^{1 / n} & =C(d(n)), \\
\sup _{d(n)} C(d(n)) & =C ; \\
\sup _{i}\left|\pi_{n i}\right|\left|p_{i} o(n)\right| & =B(n, g(n)), \\
\liminf _{n \rightarrow \infty}\{B(n, g(n))\}^{1 / \mid n-o(n)\}} & =B(g(n)), \\
\inf _{o(n)} B(g(n)) & =B .
\end{aligned}
$$

For the product set $\left\{p_{n}\right\}\left\{q_{n}\right\}$, if $b_{p q}$ is the quantity corresponding to $b$, we have

$$
b_{p q}(n, s(n))=\sup _{m, i, x}\left|\lambda_{n m}\right|\left|\pi_{m i}\right|\left|p_{i x}\right|\left|q_{x<(n)}\right|,
$$

and so on, where the $\lambda$ 's are related to $\left\{q_{n}\right\}$ as the $\pi$ 's are related to $\left\{p_{n}\right\}$.

Let $C_{v}, b_{v}, B_{v}$ stand for $C, b, B$ respectively, in case of the derived set $\left\{D^{h}\left\{p_{n}(z)\right\}\right\}$. Then we have

$$
C_{v}(n, d(n))=\sup _{i}\left\{\left|B_{n n}\right|\left|\pi_{n i}\right|\left|p_{i d(n)}\right|\left|Q_{d(n) d(n)}\right|\right\},
$$

but in view of (1), we know that

$$
\beta_{n n} \cdot Q_{d(n) d(n)}=\frac{(d(n)+1)(d(n)+2) \cdots(d(n)+h)}{(n+1)(n+2) \cdots(n+h)},
$$

then, $\lim \sup _{n \rightarrow \infty}\left\{\left|\beta_{n n}\right|\left|Q_{d(n) d(n)}\right|\right\}^{1 / n}=1$, hence

$$
C_{v}=C \text {. }
$$

Similarly,

$$
b_{v}(n, s(n))=\sup _{i}\left\{\left|B_{n n}\right|\left|\pi_{n i}\right|\left|p_{i \varepsilon^{\prime}(n)}\right|\left|Q_{8^{\prime}(n) \bullet^{\prime}(n)}\right|\right\} .
$$

Since the value of $s^{\prime}(n)$ is either equal to the value of $s(n)$ (when $s(n)$ $\geqq h$ ), or less than it (when $s(n)<h$ ), then

$$
b_{v} \leqq b \text {; }
$$


also $B_{v}(n, g(n))=\sup _{i}\left\{\left|\beta_{n n}\right|\left|\pi_{n i}\right|\left|p_{i g(n)}\right|\left|Q_{g(n) \theta(n)}\right|\right\} ;$ in view of (1), we get:

$$
\begin{array}{r}
\liminf _{n \rightarrow \infty}\left\{\left|\beta_{n n}\right|\left|Q_{o(n) o(n)}\right|\right\}^{1 /(n-g(n))=} \underset{n \rightarrow \infty}{\liminf \left\{\sigma^{\prime h}\right\} 1 /(n-o(n)) \rightarrow 1} \\
\left(\sigma^{\prime}=\lim _{n \rightarrow \infty} \frac{g(n)}{n}\right),
\end{array}
$$

then

$$
B_{v}=B \text {. }
$$

Hence in case of:

i. $a>1$, if $C \leqq 1$, the $h$ th basic derivative set is effective on $|z|=R$, where $b_{v} \leqq R \leqq B$ and $b_{v} \leqq b$.

ii. $a=1$, if $C \leqq 1$, the $h$ th basic derived set is effective on $|z|=R$, where $b_{v} \leqq R$ and $b_{v} \leqq b$.

ExAmple. Let

$$
\begin{aligned}
& p_{n}(z)=2^{n} z^{n}-\frac{1}{3^{n}} z^{2 n}-4^{n}, \\
& p_{n}(z)=z^{n},
\end{aligned}
$$

all other values of $n$,

then,

$$
\begin{aligned}
\begin{aligned}
v_{n-h}(z)= & 2^{n} n(n-1) \cdots(n-h+1) z^{n-h} \\
& -\left(1 / 3^{n}\right) 2 n(2 n-1) \cdots(2 n-h+1) z^{2 n-h}, \quad n \text { odd }>h, \\
v_{n-h}(z)= & n(n-1) \cdots(n-h+1) z^{n-h}, \quad \text { all other values of } n,
\end{aligned} \\
\begin{aligned}
& \text { i.e., } \\
& v_{n}(z)= 2^{n+h}(n+h)(n+h-1) \cdots(n+1) z^{n} \\
&-\frac{1}{3^{n+h}}(2 n+2 h) \cdots(2 n+h+1) z^{2 n+h} \\
& \quad(n \text { odd or even as } h \text { even or odd }), \\
& v_{n}(z)=(n+h)(n+h-1) \cdots(n+1) z^{n}, \\
& \quad(n \text { even or odd as } h \text { even or odd }),
\end{aligned}
\end{aligned}
$$

then

$$
z^{n}=\frac{1}{2^{n+h}(n+h) \cdots(n+1)} v_{n}(z)+\frac{1}{6^{n+h}} v_{2 n+h}(z)
$$

( $n$ odd or even as $h$ even or odd), 
$z^{n}=\frac{1}{(n+h) \cdots(n+1)} v_{n}(z) \quad(n$ even or odd as $h$ even or odd $)$

then

$$
C_{v}=1, b_{v}=0 \text {, and } B_{v}=6,
$$

i.e., the $h$ th derived set is effective in the $0 \leqq|z| \leqq 6$. Since we know also that $C=1, b=2, B=6$, i.e., the original set is effective in the annulus $2 \leqq|z| \leqq 6$, whence the result of Theorem 1 .

TheORem 2. $\left\{p_{n}(z)\right\}$ is a basic set of polynomials, such that $\lim \sup _{n \rightarrow \infty} D_{n} / n=a(a \geqq 1)$. Then its hth basic integral set is effective in the same domain of effectiveness of the original set.

Proof. By Lemma 3, we have:

$$
\left\{D^{-h}\left\{p_{n}(z)\right\}\right\} \equiv\left\{p_{n}(z)\right\}\left\{D^{-h}\left\{Q_{n}(z)\right\}\right\} .
$$

Let $C_{t}, b_{t}, B_{t}$ stand for $C, b, B$ respectively, in case of the integral set $\left\{D^{-h}\left\{p_{n}(z)\right\}\right\}$.

We know that

$$
C_{t}(n, d(n))=\sup _{i}\left\{\left|\beta_{n n}\right|\left|\pi_{n i}\right|\left|p_{i d(n)}\right|\left|Q_{d(n) d(n)}\right|\right\}
$$

but in view of (2), we get:

$\limsup _{n \rightarrow \infty}\left\{\left|\beta_{n n}\right|\left|Q_{d(n) d(n)}\right|\right\}^{1 / n}$

$$
=\limsup _{n \rightarrow \infty}\left\{\frac{(n-h+1)(n-h+2) \cdots n}{(d(n)-h+1) \cdots d(n)}\right\}^{1 / n}=1,
$$

then $C_{t}=C$.

Similarly,

$$
b_{t}=b \quad \text { and } \quad B_{t}=B
$$

Then, in case

i. If $a>1$, the $h$ th basic integral set is effective on $|z|=R$, where $b_{t} \leqq R \leqq B_{t}$ and $b_{t}=b, B_{t}=B$.

ii. If $a=1$, the $h$ th basic integral set is effective on $|z|=R$, where $b_{t} \leqq R$ and $b_{t}=b$.

Whence the result of the theorem follows.

EXAMPLE. I shall employ the set given in the previous example to determine the effectiveness of its $h$ th integral set.

In view of (2) we get: 


$$
\begin{aligned}
& t_{n+h}(z)=\frac{2^{n} z^{n+h}}{(n+1) \cdots(n+h)}-\frac{3^{-n} z^{2 n+h}}{(2 n+1) \cdots(2 n+h)}-\frac{4^{n} z^{h}}{1 \cdot 2 \cdots h} \\
& \quad(n \text { odd }), \\
& t_{n+h}(z)=\frac{z^{n+h}}{(n+1) \cdots(n+h)}
\end{aligned}
$$

then

$$
\begin{aligned}
t_{n}(z)= & \frac{2^{n-h} z^{n}}{(n-h+1) \cdots n}-\frac{3^{-(n-h)} z^{2 n-h}}{(2 n-2 h+1) \cdots(2 n-h)} \\
& -\frac{4^{n-h} z^{h}}{1 \cdot 2 \cdots h} \quad(n \text { odd or even as } h \text { even or odd }), \\
t_{n}(z)= & \frac{z^{n}}{(n-h+1) \cdots n} \quad(n \text { even or odd as } h \text { even or odd }),
\end{aligned}
$$

then

$$
\begin{aligned}
& z^{n}=\frac{1}{2^{n-h}}(n-h+1) \cdots n+ \frac{1}{6^{n-h}} t_{2 n-h}(z)+2^{n-h} t_{h}(z) \\
& \quad(n \text { odd or even as } h \text { even or odd }), \\
& z^{n}=(n-h+1) \cdots n t_{n}(z) \quad(n \text { even or odd as } h \text { even or odd }) .
\end{aligned}
$$

Hence $C_{t}=1, b_{t}=2$ and $B_{t}=6$, i.e., the $h$ th integral set is effective in the same domain of effectiveness of the original set.

THEOREM 3. $\left\{p_{n}(z)\right\}$ is a basic set of polynomials such that $\lim \sup _{n \rightarrow \infty} D_{n} / n=a(a \geqq 1)$, and of order $\omega$. Then each of its hth basic derived and integral sets is of order $\leqq \omega$.

Proof. By Lemma 2, we know:

$$
\left\{D^{h}\left\{p_{n}(z)\right\}\right\} \equiv\left\{p_{n}(z)\right\}\left\{D^{h}\left\{Q_{n}(z)\right\}\right\},
$$

and from (1),

$$
\max _{1}\left|\beta_{n i}\right|=\left|\beta_{n n}\right|=\frac{1}{(n+1) \cdots(n+h)}
$$

and

$$
\max _{1}\left|Q_{n i}\right|=\left|Q_{n n}\right|=(n+1) \cdots(n+h) .
$$

Applying Theorem 3 of [1] and since $a_{Q}=1$, we get: the order of the derivative set $\leqq \omega$. 
Similarly,

the order of the integral set $\leqq \omega$.

N.B. By this theorem we can see that:

Example. Let

$$
\text { the order of the integral set }=\omega .
$$

$$
\begin{array}{lr}
p_{n}(z)=n^{n} z^{n}-\frac{n^{2 n} z^{2 n}}{(\log n)^{n}}-n^{3 n}, & n \text { odd }>h, \\
p_{n}(z)=z^{n}, & \text { other values of } n,
\end{array}
$$

then

$$
\begin{aligned}
& v_{n-h}(z)=D^{h}\left(p_{n}(z)\right)=n^{n} n(n-1) \cdots(n-h) z^{n-h} \\
& -\frac{n^{2 n}}{(\log n)^{n}} 2 n(2 n-1) \cdots(2 n-h) z^{2 n-h}, \quad n \text { odd }>h \text {, } \\
& v_{n-h}(z)=D^{h}\left(p_{n}(z)\right)=n(n-1) \cdots(n-h) z^{n-h}, \quad \text { other values of } n \text {, } \\
& \text { i.e., } \\
& v_{n}(z)=(n+h)^{(n+h)}(n+h) \cdots n z^{n} \\
& -\frac{(n+h)^{2(n+h)}}{(\log (n+h))^{(n+h)}}(2 n+2 h) \cdots(2 n+h) z^{2 n+h}
\end{aligned}
$$

Applying Theorem 1 of [1] we find $\omega_{v}=1$. Since $\omega=2$, then $\omega_{v}<\omega$. We can find easily, also, that the order of the integral set is 2 , i.e., $\omega_{t}=\omega$.

THEOREM 4. $\left\{p_{n}(z)\right\}$ is a basic set of polynomials, such that $D_{n}$ $=O\left(n^{a}\right)(a \geqq 1)$, and of order $\omega$. Then its hth basic derived set is of order $\leqq \omega$.

Proof. Here I do not use the product of $\left\{p_{n}(z)\right\}$ and $\left\{Q_{n}(z)\right\}$, but obtain the result directly. I shall start by proving that the order of the first derived set is $\leqq \omega$.

Since $z^{n+1}=\sum_{i} \pi_{n+1 \cdot i} p_{i}(z)=\sum_{i} \pi_{n+1 \cdot i} \sum_{j=0} p_{i j} z$, by differentiating we get:

$$
\begin{aligned}
\left|(n+1) z^{n}\right| & =\left|\sum_{i=0}^{j} \pi_{n+1 \cdot i} \sum_{j=1}^{j} j p_{i j} z^{j-1}\right| \\
& \leqq D_{n+1} \sum_{i=0}^{j}\left|\pi_{n+1 \cdot i}\right| \sum_{j=1}^{j}\left|p_{i j} z^{i-1}\right| \quad(|z|>1),
\end{aligned}
$$


then

$$
\begin{aligned}
\left\{\omega_{n}(R)\right\}_{v} & \leqq \frac{D_{n+1}}{n+1} \sum_{i=0}^{j}\left|\pi_{n+1 \cdot i}\right| \sum_{j=1}^{j}\left|p_{i j}\right| R^{i} \\
& \leqq \frac{D_{n+1}}{n+1} \sum_{i=0}^{i}\left|\pi_{n+1 \cdot i}\right| \sum_{j=0}^{j}\left|p_{i j}\right| R^{i} \\
& \leqq \frac{D_{n+1}}{n+1}\left(\omega_{n}(R)\right) .
\end{aligned}
$$

Then,

$$
\lim _{R \rightarrow \infty} \limsup _{n \rightarrow \infty} \frac{\log \left(\omega_{n}(R)\right)_{0}}{n \log n} \leqq \lim _{R \rightarrow \infty} \limsup _{n \rightarrow \infty} \frac{\log D_{n+1}+\log \left(\omega_{n}(R)\right)}{n \log n},
$$

in view of the definition of order $[2 ; 3]$, we get:

the order of the derivative set $\leqq \omega$.

By applying this theorem to the second derived set, we find that the order of the second derived set is less than or equal to the order of the first derived set, i.e., the order of the second derived set is less than or equal to $\omega$. By proceeding in this way, we get the required result.

TheOREM 5. If $\left\{p_{n}(z)\right\}$ is a basic set of polynomials of order $\omega$, then its hth basic integral set is of order $\leqq \omega$.

Proof. Here, again, I use a direct proof.

$$
z^{n-1}=\sum_{i=0} \pi_{n-1 \cdot i} p_{i}(z)=\sum_{i=0} \pi_{n-1 \cdot i} \sum_{j=0} p_{i j} z^{i}
$$

By integrating, we get:

$$
\frac{z^{n}}{n}=\sum_{i=0} \pi_{n-1 \cdot i} \sum_{j=0} \frac{1}{j+1} p_{i j}\left(z^{j+1}-c\right) \quad(c \text { constant }),
$$

then

$$
\left|z^{n}\right| \leqq n \sum_{i=0}\left|\pi_{n-1 \cdot i}\right| \sum_{j=0}\left|p_{i j} z^{j+1}\right|, \quad|z|>1,
$$

then

$$
\left(\omega_{n}(R)\right)_{t} \leqq n \sum_{i=0}\left|\pi_{n-1 \cdot i}\right| \sum_{j=0}\left|p_{i j}\right| R^{j+1}=n R\left(\omega_{n-1}(R)\right)
$$

then 


$$
\begin{aligned}
\lim _{R \rightarrow \infty} \underset{n \rightarrow \infty}{\limsup } \frac{\log \left(\omega_{n}(R)\right)_{t}}{n \log n} & \\
& \leqq \lim _{R \rightarrow \infty} \limsup _{n \rightarrow \infty}\left\{\frac{\log n+\log R+\log \omega_{n-1}(R)}{n \log n}\right\} \\
& \leqq \lim _{R \rightarrow \infty} \limsup _{n \rightarrow \infty} \frac{\log \omega_{n-1}(R)}{n \log n}=\omega .
\end{aligned}
$$

By proceeding in this way to the other integrals, we get the required result of the theorem.

EXAMPLE. I give this example to show that the condition $D_{n}=O\left(n^{a}\right)$ is necessary for the truth of Theorem 4, but not necessary in case of integral sets, i.e., in Theorem 5 .

Let

$$
\begin{array}{ll}
p_{n}(z)=n^{n} z^{n}-\frac{n^{2 n}\left(z^{2 n}\right)^{2 n}}{(\log n)^{2 n}}-n^{3 n}, & n \text { odd, } \\
p_{n}(z)=z^{n}, & n \text { even or } 0 .
\end{array}
$$

I shall be satisfied to deal with the first derived and integral sets. Applying the definition of order, we get:

$$
\omega=2, \quad \omega_{v}=3, \quad \omega_{t}=2,
$$

hence $\omega_{t}=\omega$, i.e., Theorem 5 is verified and $\omega_{v}>\omega$, i.e., Theorem 4 is not verified.

\section{REFERENCES}

1. M. N. Mikhail, Basic sets of polynomials and their reciprocal, product and quotient sets, Duke Math. J. (to appear).

2. J. M. Whittaker, Interpolatory function theory, Cambridge, 1935.

3. - Sur les séries de base de polynomes quelconques, Paris, 1949.

Birkbeck College, University of London 